\title{
Difficulties in diagnosing terminal ileitis due to Yersinia pseudotuberculosis
}

\author{
H. F. Wunderink • P. M. Oostvogel • I. H. M. E. Frénay • \\ D. W. Notermans • A. Fruth • E. J. Kuijper
}

Received: 5 June 2013 / Accepted: 24 July 2013

(C) Springer-Verlag Berlin Heidelberg 2013

\begin{abstract}
We report three patients with terminal ileitis and positive fecal cultures with Yersinia pseudotuberculosis. From one patient, a virulence plasmid (pYV)-negative Y. pseudotuberculosis was isolated, which represents the second finding of a pYV-negative isolate associated with human disease. All patients were treated with ciprofloxacin and fully recovered. Since conventional culture methods for yersiniosis are gradually replaced with molecular tests not recognizing $Y$. pseudotuberculosis, we recommend to include a specific culture medium or to apply a specific polymerase chain reaction (PCR) assay on fecal samples from patients suspected of terminal ileitis.
\end{abstract}

\section{Introduction}

The genus Yersinia belongs to the family of Enterobacteriaceae and is composed of 11 species, of which only Y. pestis, Y. pseudotuberculosis, and some $Y$. enterocolitica serotypes are

\section{H. F. Wunderink $(\bowtie) \cdot$ E. J. Kuijper}

Department of Medical Microbiology, Leiden University Medical Center, E1-200, Albinusdreef 2, 2333 ZA Leiden, The Netherlands e-mail: h.f.wunderink@lumc.nl

P. M. Oostvogel

Department of Clinical Microbiology, Medical Center Haaglanden (MCH), The Hague, The Netherlands

\section{H. M. E. Frénay}

Regional Laboratory for Medical Microbiology and Infectious Diseases, Dordrecht-Gorinchem and Albert Schweitzer Hospital, Dordrecht, The Netherlands

\section{W. Notermans}

Laboratory for Infectious Diseases and Screening, National Institute for Public Health and the Environment, Bilthoven, The Netherlands

\section{A. Fruth}

National Reference Center for Salmonella and Other Bacterial Enteric Pathogens, Robert Koch Institute, Wernigerode, Germany human pathogens [1-3]. While Y. pestis is known as the causative agent of the plague and causes no gastrointestinal disease, Y. enterocolitica and Y. pseudotuberculosis are known as causative agents of gastrointestinal disease [1-4].

Y. pseudotuberculosis is a zoonotic pathogen transmitted by the fecal-oral route that has been recovered from various rodents, rabbits, deer, bats, farm animals, and wild and domestic birds [2, 3, 5, 6]. In 1883, Knapp and Masshoff were the first to isolate $Y$. pseudotuberculosis from a mesenteric lymph node of a patient with a clinical syndrome of acute appendicitis [7]. After an incubation time of 3-7 days, Y. pseudotuberculosis may cause mucosal ulceration in the terminal ileum and, sometimes, the adjacent colon, lesions in Peyer's patches, and enlargement of mesenteric lymph nodes [8, 9]. In severe cases, septicemia, thrombosis of mesenteric vessels, focal abscesses, intestinal necrosis, and hemorrhage may occur. Predisposing host factors for a severe course are diabetes mellitus, liver cirrhosis, and hemochromatosis $[10,11]$. Post-infectious complications such as reactive (poly)arthritis and erythema nodosum can result from an immunological reaction within several weeks [2].

Y. pseudotuberculosis is a pleomorphic, facultative anaerobic, non-spore-forming, Gram-negative rod that grows at temperatures ranging from 4 to $43{ }^{\circ} \mathrm{C}$, with the optimal temperature being between 25 and $28^{\circ} \mathrm{C}$. Y. pseudotuberculosis grows on most routine media, including MacConkey, blood, and chocolate agar, incubated at $35^{\circ} \mathrm{C}$ in ambient air, but may be overgrown by other enteric bacteria in clinical samples. Therefore, a selective agar, cefsulodin-irgasan-novobiocin (CIN) agar, incubated at $25-28{ }^{\circ} \mathrm{C}$, is used for the isolation of $Y$. pseudotuberculosis from fecal samples [12-14]. $\mathrm{CIN}$ agar contains D-mannitol and fermentation of this carbohydrate in the presence of neutral red produces characteristic red colonies. Other bacteria, such as Plesiomonas and Aeromonas species, also grow on CIN agar, but these can be differentiated from Yersinia species by their positive oxidase test. Y. pseudotuberculosis can be serotyped based on O- 
somatic antigen, which is the most distal component of the lipopolysaccharide membrane. Twenty-one serotypes have been identified, with $\mathrm{O}$ Group I (O:1) accounting for most human cases and sometimes $0: 2$ or O:3 $[2,15]$. The virulence of $Y$. pseudotuberculosis is associated with the presence of multiple virulence markers, including the plasmid of Yersinia virulence (pYV), the invasion adhesion molecule (invA) gene, the attachment invasion locus (ail) gene, and the high pathogenicity island (HPI) [1-3, 16-19].

We report three patients with terminal ileitis due to $Y$. pseudotuberculosis diagnosed by conventional culture.

\section{Case reports}

Case 1

A previously healthy 24-year-old female was admitted to a local hospital with progressive right lower quadrant abdominal pain, suspected of appendicitis. There was no diarrhea and temperature was $37.7^{\circ} \mathrm{C}$. On physical examination, there was pain on percussion and rebound tenderness of the right lower abdominal quadrant. Laboratory examinations showed a leukocytosis (total white cell count $12.1 \times 10^{9} / 1$ ), an elevated C-reactive protein level $(49 \mathrm{mg} / \mathrm{l})$, and no other abnormalities. A pregnancy test was negative and urine sediment was normal. A computed tomography (CT) scan showed a normal appendix, significant thickening of the terminal ileum wall over a length of $7 \mathrm{~cm}$, thickening of the adjacent cecum wall, enlarged mesenteric lymph nodes, and no free intraperitoneal fluid.

Morbus Crohn was suspected, and prednisone and azathioprine were started. A colonoscopy was performed and revealed a swollen mucous membrane with little ulceration of the terminal ileum and adjacent cecum. Biopsies were performed and pathological examination showed no signs of M. Crohn or idiopathic colitis. To exclude an infectious origin, a fecal culture was performed. The fecal culture revealed growth of Y. pseudotuberculosis on CIN medium. Prednisone and azathioprine were stopped and the patient was treated with ciprofloxacin $500 \mathrm{mg}$ twice daily for 5 days. At follow up 2 months later, there were no complaints, laboratory examinations were normal, and a magnetic resonance imaging (MRI) enterography showed no abnormalities.

\section{Case 2}

A previously healthy 17-year-old female presented to the emergency department with progressive right lower quadrant abdominal pain since 5 days, suspected of appendicitis. There was no diarrhea and temperature was $38.3{ }^{\circ} \mathrm{C}$. On physical examination, there was rebound tenderness of the right lower abdominal quadrant. Laboratory examinations showed a leukocytosis (total white cell count $14 \times 10^{9} / 1$ ), an elevated C-reactive protein level $(54 \mathrm{mg} / \mathrm{l})$, an elevated erythrocyte sedimentation rate $(29 \mathrm{~mm} / \mathrm{h})$, and no other abnormalities. A pregnancy test was negative and urine sediment was normal. An abdominal ultrasonography could not visualize the appendix, but showed enlarged mesenteric lymph nodes. A CT scan showed a normal appendix, significant thickening of the terminal ileum wall over a length of $10 \mathrm{~cm}$, enlarged mesenteric lymph nodes, and no free intraperitoneal fluid (Fig. 1).

A terminal ileitis was suspected, with a differential diagnosis of M. Crohn and an infectious ileitis. Two fecal cultures were performed and both grew Y. pseudotuberculosis on CIN medium. The patient was treated with ciprofloxacin $500 \mathrm{mg}$ twice daily for 5 days. At follow up 2 months later, there were no complaints, laboratory examinations were normal, and abdominal ultrasonography showed no abnormalities.

\section{Case 3}

A previously healthy 33-year-old female presented to the emergency department of a local hospital with progressive right lower quadrant abdominal pain since 7 days, suspected of appendicitis. There was no diarrhea and temperature was $37.3^{\circ} \mathrm{C}$. On physical examination, there was rebound tenderness of the right lower abdominal quadrant. Laboratory examinations showed normal leukocytes (total white cell count $\left.7.9 \times 10^{9} / \mathrm{l}\right)$, and an elevated C-reactive protein level $(31 \mathrm{mg} / \mathrm{l})$. Urine sediment was normal. An abdominal ultrasonography showed thickening of the terminal ileum atypical for $\mathrm{M}$. Crohn and enlarged mesenteric lymph nodes. No CT scan was performed.

An infectious terminal ileitis was suspected and a fecal culture was performed. Prior to final diagnosis, treatment was started with ciprofloxacin $500 \mathrm{mg}$ twice daily for 5 days. The fecal culture revealed growth of $Y$. pseudotuberculosis on CIN medium. At follow up 1 month later, there were no complaints

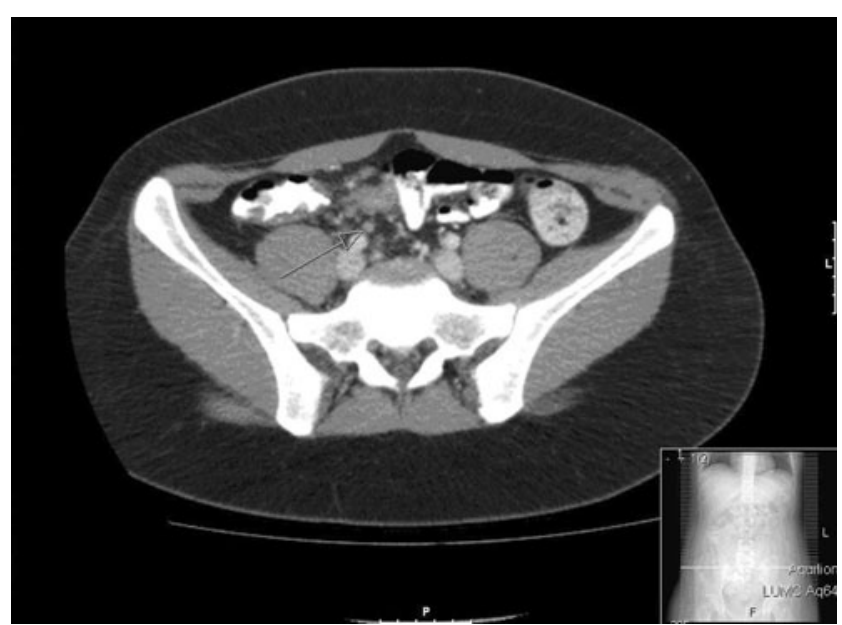

Fig. 1 Computed tomography (CT) scan of case 2, showing an enlarged mesenteric lymph node (arrow) 
and laboratory examinations were normal. No control ultrasound or CT scan was performed.

\section{Results}

Because of the clinical suspicion of an infectious terminal ileitis in all three cases, fecal samples were incubated on $\mathrm{CIN}$ agar at $25-28^{\circ} \mathrm{C}$, for the isolation of Yersinia species. The cultured isolates were then identified as Y. pseudotuberculosis by matrix-assisted laser desorption ionization time-offlight (MALDI-TOF) mass spectrometry (MS) (Bruker Daltonik GmbH, Bremen, Germany) [20, 21]. Further characterization of the isolated $Y$. pseudotuberculosis strains was performed by serotyping and molecular techniques. Two isolates belonged to serotype $\mathrm{O} 1$, the most frequently found type in human infections and one isolate was not serotyped $[2,3]$. Y. enterocolitica $16 \mathrm{~S}$ rRNA polymerase chain reaction (PCR), performed at the National Institute for Public Health and the Environment (RIVM, Bilthoven, the Netherlands) and the Robert Koch institute (RKI, Wernigerode, Germany), was negative in two isolates and was not performed for one isolate. $Y$. enterocolitica ail gene PCR, performed at the RKI, was negative in all three isolates. No diagnostics were performed for the Y. pseudotuberculosis chromosomal invA and ail gene or the HPI, because these were not available at the RIVM and RKI. Two isolates were positive in the Y. pseudotuberculosis pYV PCR, performed at the RKI, and one isolate was negative. Susceptibility of the $Y$. pseudotuberculosis was determined with Etest (AB Biodisk, Solna, Sweden) and performed for amoxicillin [minimum inhibitory concentration (MIC) $0.25-0.5 \mu \mathrm{g} / \mathrm{ml}$ ], cefuroxime (MIC $0.5-0.75 \mu \mathrm{g} / \mathrm{ml}$ ), chloramphenicol (MIC 1.5-3 $\mu \mathrm{g} / \mathrm{ml}$ ), ciprofloxacin (MIC 0.023$0.047 \mu \mathrm{g} / \mathrm{ml}$ ), and tetracycline (MIC $0.75-1.5 \mu \mathrm{g} / \mathrm{ml}$ ).

\section{Discussion}

In 2012, three unrelated patients were diagnosed with $Y$. pseudotuberculosis-associated terminal ileitis in different hospitals in the Netherlands. The patients were female, between 17 and 33 years of age, and presented with progressive right lower abdominal pain and fever. None of the patients had underlying disease. Remarkably, radiological results were the first indication of terminal ileitis in all patients. All patients fully recovered after treatment with ciprofloxacin without any complication.

The virulence of Y. pseudotuberculosis is associated with the presence of a genetically conserved virulence plasmid, termed pYV. It is thought that loss of pYV leads to avirulence $[1,2$, 22]. pYV carries components of a type three secretion system, which forms a needle structure on the surface of Y. pseudotuberculosis that interacts with target cells and enables the injection of at least six different Yersinia outer membrane proteins (Yops) [1, 2, 22, 23]. Yops disrupt the primary immune response and adaptive inflammatory cascade, and, in this way, prevent phagocytosis $[2,3,22,23]$. Besides plasmid-encoded genes, Y. pseudotuberculosis also have chromosomal-encoded virulence markers, like the invA and ail genes, and some strains harbor the HPI. The invA gene encodes for an epithelial cell adhesion molecule, which facilitates efficient binding to intestinal mucosal cells and translocation from the lumen to Peyer's patches. The ail gene encodes for an outer membrane protein that mediates complement resistance; its nucleotides composition differs slightly between Y. enterocolitica and Y. pseudotuberculosis $[18,19,24,25]$. The HPI encodes an iron uptake system for the siderophore yersiniabactin and its presence makes a strain highly virulent. HPI plays a key role in the systemic spread of Yersinia, by which infection of the spleen and liver can occur $[3,16]$.

The absence of pYV in an isolate causing terminal ileitis (case 1) is in contradiction with the common hypothesis that the absence of pYV leads to avirulence of the bacteria $[1,2$, 22]. However, it is reported in the literature that $Y$. pseudotuberculosis can lose $\mathrm{pYV}$ after subculture and storage, in particular, at $37^{\circ} \mathrm{C}$ [18]. Consequently, the pYV is not an ideal DNA target for the detection of pathogenic Yersinia strains, and it is better to combine it with the detection of a chromosomal virulence gene [17]. Animal infection studies showed that pYV-negative $Y$. pseudotuberculosis strains are able to establish intestinal infections and even colonize the Peyer's patches and mesenteric lymph nodes, showing that the presence of pYV is not required for the entry of Yersinia into host tissues [3, 26]. For instance, Fukushima et al. reported a case of a Japanese girl with a mesenteric lymphadenitis caused by a pYV-negative $Y$. pseudotuberculosis that was positive for the chromosomal virulence markers [26]. So, although the loss of pYV leads to a significant decrease in the level of virulence, it seems likely that, also, pYV-negative strains can cause human infection $[3,26]$.

Although the reported incidence of human infections due to Y. pseudotuberculosis is low, this may be due to the fact that CIN agar is not systematically used for the culture of Yersinia spp. in diarrheal patients. Moreover, most of the molecular diagnostic techniques use the $Y$. enterocolitica ail gene as the target, which is not present in Y. pseudotuberculosis [24, 27, 28]. This means that selective culture techniques or the performance of a specific $Y$. pseudotuberculosis PCR are warranted when $Y$. pseudotuberculosis is suspected, and also when diarrhea is not present. We also want to encourage the development of $Y$. pseudotuberculosis-specific PCRs for the combined detection of pYV and chromosomal-encoded genes in the light of the pYV-negative cultured Y. pseudotuberculosis isolate.

Conflict of interest The authors declare that they have no conflict of interest. 


\section{References}

1. Carniel E, Mollaret HH (1990) Yersiniosis. Comp Immunol Microbiol Infect Dis 13:51-58

2. Bockemühl J, Roggentin P (2004) Intestinal yersiniosis. Clinical importance, epidemiology, diagnosis, and prevention. Bundesgesundheitsblatt Gesundheitsforschung Gesundheitsschutz 47:685-691

3. Viboud GI, Bliska JB (2005) Yersinia outer proteins: role in modulation of host cell signaling responses and pathogenesis. Annu Rev Microbiol 59:69-89

4. Ijichi S, Kusaka T, Okada H, Fujisawa T, Kobara H, Itoh S (2012) Terminal Ileitis caused by Yersinia pseudotuberculosis mimicking Crohn disease in childhood. J Pediatr Gastroenterol Nutr 55:e125

5. Martínez PO, Fredriksson-Ahomaa M, Pallotti A, Rosmini R, Houf K, Korkeala H (2011) Variation in the prevalence of enteropathogenic Yersinia in slaughter pigs from Belgium, Italy, and Spain. Foodborne Pathog Dis 8:445-450

6. Muhldorfer K, Wibbelt G, Haensel J, Riehm J, Speck S (2010) Yersinia species isolated from bats, Germany. Emerg Infect Dis 16:578-580

7. Knapp W, Masshoff W (1954) Etiology of suppurative reticulocytic lymphadenitis, a practically important disease frequently simulating acute appendicitis. Dtsch Med Wochenschr 79:1266-1271

8. El-Maraghi NR, Mair NS (1979) The histopathology of enteric infection with Yersinia pseudotuberculosis. Am J Clin Pathol 71:631-639

9. Kühn H, Freissler G (1983) Pathogenesis and pathologic anatomy of human Yersinia infections. Leber Magen Darm 13:270-277

10. Deacon AG, Hay A, Duncan J (2003) Septicemia due to Yersinia pseudotuberculosis - a case report. Clin Microbiol Infect 9:1118-1119

11. Kaasch AJ, Dinter J, Goeser T, Plum G, Seifert H (2012) Yersinia pseudotuberculosis bloodstream infection and septic arthritis: case report and review of the literature. Infection 40:185-190

12. Schiemann DA (1979) Synthesis of a selective agar medium for Yersinia enterocolitica. Can J Microbiol 25:1298-1304

13. Head CB, Whitty DA, Ratnam S (1982) Comparative study of selective media for recovery of Yersinia enterocolitica. J Clin Microbiol 16:615-621

14. Savin C, Leclercq A, Carniel E (2012) Evaluation of a single procedure allowing the isolation of enteropathogenic Yersinia along with other bacterial enteropathogens from human stools. PLoS One 7:e41176

15. De Castro C, Kenyon JJ, Cunneen MM, Molinaro A, Holst O, Skurnik M, Reeves PR (2013) The O-specific polysaccharide structure and gene cluster of serotype O:12 of the Yersinia pseudotuberculosis complex, and the identification of a novel L-quinovose biosynthesis gene. Glycobiology 23:346-353

16. Eppinger M, Rosovitz MJ, Fricke WF, Rasko DA, Kokorina G, Fayolle C, Lindler LE, Carniel E, Ravel J (2007) The complete genome sequence of Yersinia pseudotuberculosis IP31758, the causative agent of Far East scarlet-like fever. PLoS Genet 3:e142

17. Fukushima H, Shimizu S, Inatsu Y (2011) Yersinia enterocolitica and Yersinia pseudotuberculosis detection in foods. J Pathog 2011:735308

18. Ho DK, Riva R, Skurnik M, Meri S (2012) The Yersinia pseudotuberculosis outer membrane protein Ail recruits the human complement regulatory protein factor H. J Immunol 189:3593-3599

19. Ho DK, Riva R, Kirjavainen V, Jarva H, Ginström E, Blom AM, Skurnik M, Meri S (2012) Functional recruitment of the human complement inhibitor C4BP to Yersinia pseudotuberculosis outer membrane protein Ail. J Immunol 188:4450-4459

20. Ayyadurai S, Flaudrops C, Raoult D, Drancourt M (2010) Rapid identification and typing of Yersinia pestis and other Yersinia species by matrix-assisted laser desorption/ionization time-of-flight (MALDI-TOF) mass spectrometry. BMC Microbiol 10:285

21. Lasch P, Drevinek M, Nattermann H, Grunow R, Stämmler M, Dieckmann R, Schwecke T, Naumann D (2010) Characterization of Yersinia using MALDI-TOF mass spectrometry and chemometrics. Anal Chem 82:8464-8475

22. Shao F (2008) Biochemical functions of Yersinia type III effectors. Curr Opin Microbiol 11:21-29

23. Matsumoto H, Young GM (2009) Translocated effectors of Yersinia. Curr Opin Microbiol 12:94-100

24. Lambertz ST, Nilsson C, Hallanvuo S (2008) TaqMan-based realtime PCR method for detection of Yersinia pseudotuberculosis in food. Appl Environ Microbiol 74:6465-6469

25. Lambertz ST, Nilsson C, Hallanvuo S, Lindblad M (2008) Real-time PCR method for detection of pathogenic Yersinia enterocolitica in food. Appl Environ Microbiol 74:6060-6067

26. Fukushima H, Sato T, Nagasako R, Takeda I (1991) Acute mesenteric lymphadenitis due to Yersinia pseudotuberculosis lacking a virulence plasmid. J Clin Microbiol 29:1271-1275

27. Thoerner P, Bin Kingombe CI, Bögli-Stuber K, Bissig-Choisat B, Wassenaar TM, Frey J, Jemmi T (2003) PCR detection of virulence genes in Yersinia enterocolitica and Yersinia pseudotuberculosis and investigation of virulence gene distribution. Appl Environ Microbiol 69:1810-1816

28. Wannet WJ, Reessink M, Brunings HA, Maas HM (2001) Detection of pathogenic Yersinia enterocolitica by a rapid and sensitive duplex PCR assay. J Clin Microbiol 39:4483-4486 